\title{
Minderheitenschutz für Spezialisten!
}

A 11 e rgien gehören zu den großen gesundheitlichen Herausforderungen unserer Gesellschaft. Darüber sind sich alle einig. Es wird viel geredet über Prävention, Handlungsbedarf und Maßnahmen, es wird auch viel geschrieben; es gibt Leitlinien, Richtlinien, Qualitätssicherungskonzepte, Stellungnahmen, Positionspapiere und vieles mehr. Getan wird leider wenig.

Es gibt Ärzte, die sich dieser Herausforderung stellen, die sich nach Absolvierung einer Facharztweiterbildung in einem organbezogenen Gebiet noch zwei Jahre speziell die Kenntnisse erarbeiten, um als ,Allergologen“ betroffene Patienten besser behandeln zu können als dies bislang häufig der Fall war. Durch die gesundheitspolitischen Entwicklungen der letzten Zeit droht nun die Gefahr, daß diesen Kollegen ihre Arbeit unmöglich gemacht wird. Wenn die Allergiediagnostik mehr kostet als einbringt, wird sie nicht mehr durchgeführt werden. Eine Zeit lang werden sich noch einige Idealisten den Luxus leisten, aus früheren Überschüssen die jetzt nötige Diagnostik und Therapie für ihre Patienten zu bezahlen. Schon bald wird das aber aufhören.

Leider stehen wir in Deutschland nicht allein; in vielen Ländern gibt es ähnliche Probleme. Die Kostensteigerungen im Gesundheitswesen versucht man mit relativ einfallslosen Mitteln zu bekämpfen. Auch imWunderland USA, wo der Allergologe ein eigener Facharzt ist, ist die Situation alles andere als rosig. Zwischen „Health Management Organisation“ (HMO), Global-Budget und „Gatekeeper”-Doktor drohen die Spezialisten genauso aufgerieben zu werden wie bei uns durch die Trends zum Primärarztsystem.

Viele vernünftige Politiker sehen dies durchaus, verweisen jedoch auf die standespolitischen Regeln der Selbstverwaltung, wo in den verschiedenen Vereinigungen und Ausschüssen die lebens- oder ,,todeswichtigen“" Entscheidungen getroffen werden - meist auf dem Rücken der nicht anwesenden
Minderheiten. Da wird ganz demokratisch abgestimmt. Sachliche Arg u m e nte und Stellungnahmen werden - gelesen oder ungelesen - in geduldigen Leitzordnern abgelegt, wie z.B. das nun schon fast drei Jahre vorliegende Konzept zur „Qualitätssicherung der allergologischen Versorgung in Deutschland". Dieses Papier verschwand nach Verabschiedung durch Vertreter von acht Gesellschaften in einem echten Konsens schließlich kurz vor der Verabschiedung einfach in der Schublade, wo es seither darauf wartet, auf eine Tagesordnung des Ärztekammervorstandes gesetzt zu werden.

Ende Februar 1999 waren die Präsidenten von DGAI und ÄDA gemeinsam zu einem Gespräch im Bundesgesundheitsministerium, wo wir unsere Anliegen detailliert vorbrachten (siehe auch ÄDA-Mitteilungen in diesem Heft). Wir haben dort ganz klare Forderungen gestellt und der Hoffnung Ausdruck verliehen, daß - wenn schon alles neu geregelt wird - vielleicht auch einmal etwas zum Besseren geregelt werden könnte, etwa im Sinne eines „Minderheitenschutzes“ für Spezialisten oder der Verpflichtung zur Einbringung von Sachverstand in bestimmte Ausschüsse.

Schließlich haben wir die Ve r t r e t e r des Ministeriums darauf hingewiesen, daß das Bundesgesundheitsministerium als oberste Aufsichtsbehörde Sorg e dafür zu tragen hat, daß Patienten in Deutschland dem Wissenstand gemäß ordentlich versorgt werden. Wir haben die Befürchtung geäußert, daß durch eine Verschleppung der Diagnosestellung und der damit verbundenen Hinauszögerung einer rechtzeitigen Therapieeinleitung sich die Krankheiten verschlimmern können - bis hin zum töd-

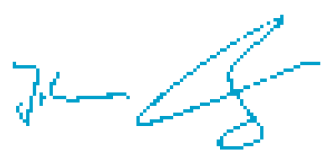

Prof. Dr. Dr. J. Ring lichen Ausgang. Dies kann auch in Deutschland jeden Tag passieren. Wi r haben auch darauf hingewiesen, daß sich hieraus eine Situation ergeben könnte, wie sie im Zusammenhang mit unsachgemäß produzierten Blutkonserven zu Beginn der AIDS-Ära in manchen Ländern entstanden ist.

Aber wir haben nicht nur gewarnt, sondern auch Vorschläge unterbreitet, die das Problem der Qualitätssicherung zugleich mit dem der Kostendämpfung einer Lösung näher bringen können. Der erste Schritt in diese Richtung ist die Umsetzung der oben genannten „Qualitätsrichtlinien“. Früher konnten wir sagen: Die beste Standespolitik ist es, die Patienten gut zu behandeln. Heute müssen wir darum kämpfen, daß dies überhaupt möglich ist.

Es steht außer Zweifel, daß allerg ische Erkrankungen in dramatischer Weise zugenommen haben. Dem gegenüber steht eine drastische Abnahme der Möglichkeiten, allergologische Leistungen überhaupt erbringen $\mathrm{zu}$ können. Diese Schere kann der einzelne Allergologe nicht mehr schließen; es wird zu einem spürbaren Qualitätsverlust in der Versorgung kommen. Wir als Berufsverband oder wissenschaftliche Fachgesellschaft haben die Aufgabe, immer wieder auf diese brennende Problematik hinzuweisen.

Es fehlt nicht an guten und willigen Ärzten, auch nicht an kreativen und e n e rgischen Forschern - wie das gerade die DGAI-Frühjahrstagung in Mainz wieder gezeigt hat - es fehlt nicht an interdisziplinärem Austausch, es fehlt nicht an neuen Ideen und Konzepten, die große Hoffnung für viele $\mathrm{B}$ e $\mathrm{t} \mathrm{r}$ o ffene beinhalten! Wir dürfen uns durch den politischen Gegenwind nicht aus der Fahrt bringen lassen.

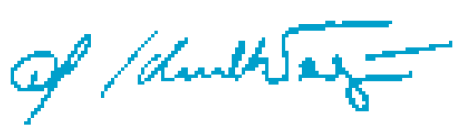

Prof. Dr. G. Schultze-Werninghaus 